\title{
Kritik und Normativität in der (empirischen) Legitimitätsforschung
}

\author{
Christiane Barnickel (iD)
}

Eingegangen: 26. Juli 2019 / Überarbeitet: 24. Oktober 2020 / Angenommen: 5. November 2020 / Online publiziert: 7. Dezember 2020

(C) Der/die Autor(en) 2020

Zusammenfassung Der Beitrag nimmt die seit längerer Zeit in der Legitimitätsforschung geführte Debatte um die als zu reduktionistisch empfundene, dichotome Unterscheidung zwischen normativen und empirischen Zugängen zum Ausgangspunkt, um aufzuzeigen, welche unterschiedlichen normativen Dimensionen auch der empirischen Legitimitätsforschung inhärent sind. Hierzu wird eine Systematisierung vorgeschlagen, die die Debattenstränge zu Normativität und Kritik sowie die bewertungssoziologische Unterscheidung zwischen Werten und Bewerten aufnimmt. Es wird argumentiert, dass durch diese Differenzierungen unterschiedliche Lagerungen der Legitimationsforschung, insbesondere der empirischen Legitimationsforschung, greifbar werden.

Hierzu extrahiert der Beitrag zwei Leitunterscheidungen zwischen, erstens, Werten und Bewerten sowie, zweitens, danach, wer jeweils wertet und bewertet. Entlang dieser Achsen ordnet der Beitrag exemplarisch Zugriffe der bestehenden Legitimitäts- und Legitimationsforschung, auf der Basis wessen normativen Referenzrahmens ein Legitimitätsurteil gefällt wird und auf welcher Ebene Legitimität beurteilt wird, ein. So wird illustriert, dass Unterschiede in der normativen Lagerung anhand der Systematisierung klarer zutage treten und unterschiedliche Spielarten der Legitimationsforschung genauer verortet werden können als in bisherigen Systematisierungsversuchen.

Dabei geht es dem Beitrag nicht darum, für ein bestimmtes Modell der Legitimitätsforschung zu plädieren. Vielmehr soll die Vielfalt des Umgangs mit der normativen Dimension - und damit auch der (sich ergänzenden) Varianten des Verständnisses von Legitimität - in der empirischen Forschung aufgefächert werden. So wird veranschaulicht, auf welchen Ebenen Kritik und Normativität jeweils ver-

C. Barnickel $(\square)$

Kulturwissenschaftliche Fakultät, Lehrstuhl für Europa-Studien, Europa-Universität Viadrina, Große Scharrnstr. 59, 15230 Frankfurt (Oder), Deutschland

E-Mail: barnickel@europa-uni.de 
ortet sind, um die Implikationen für eine (meta-)kritische Legitimitätsforschung zu diskutieren.

Schlüsselwörter Legitimation · Demokratie · Bewertungssoziologie · Kritikbegriffe · Politikwissenschaft

\title{
Critique and Normativity in (Empirical) Legitimacy Research
}

\begin{abstract}
This article takes the long-standing debate within legitimacy research about the dichotomic differentiation between normative and empirical approaches, which is perceived to be too reductionist, as a starting point to identify different normative dimensions inherent in empirical legitimacy research. For this purpose, a systematization is suggested that draws upon the discussion on normativity and critique as well as on the differentiation between valuation and evaluation as suggested by (sociological) valuation studies. It is argued that these distinctions help make different emphases in legitimation research more precise, particularly in empirical legitimation research.
\end{abstract}

The article extracts two guiding differences between, first, valuation and evaluation and, second, between who valuates and evaluates. Along these axes, the text classifies approaches in legitimacy and legitimation research on the basis of whose normative frame of reference guides the judgment of legitimacy and at which level legitimacy is evaluated. This illustrates that the systematization suggested illuminates differences with respect to the normative dimension within legitimation research and helps to locate them properly.

The article does not intend to advocate a specific approach to legitimation research. Rather, it aims to underline the plurality in dealing with the normative dimension - and thus (complementary) varieties of understandings of legitimacy-in empirical research. This demonstrates where critique and normativity are situated in order to discuss the implications for (meta)critical legitimacy research.

Keywords Legitimation - Democracy - Sociology of valuation and evaluation • Concepts of critique $\cdot$ Political science

\section{Einleitung}

Politikwissenschaftliche Annäherungen an Legitimitätsfragen werden häufig in einen normativen und einen empirischen Strang unterteilt. Bei normativen Zugriffen steht die Frage nach der „Anerkennungswürdigkeit“ (Habermas 1976, S. 39) von (nationaler, internationaler oder supranationaler) Herrschaft im Zentrum. Häufig, aber beileibe nicht immer, sind die normativen Positionen, die die Kriterien der Rechtfertigbarkeit etablieren, aus - ihrerseits pluralen - demokratietheoretischen Konzeptionen abgeleitet (vgl. beispielsweise Barker 2007; Abromeit und Stoiber 2007; Kriesi 2013). Der empirische Strang der Legitimitätsforschung interessiert sich für die tatsächliche Anerkennung oder Akzeptanz und geht nicht zuletzt auf Max Weber zurück, der die Orientierung des Handelns an der „Vorstellung vom Beste- 
hen einer legitimen Ordnung“, am „Legitimitäts-Glauben“ (beide Zitate bei Weber 1980[1921], S. 16), betonte.

Trotz dieser (vordergründigen) Arbeitsteilung sind normative und empirische Perspektiven verknüpft (vgl. Dammayr et al. 2015, S. 13): So kann die empirische „Realität“" gegenüber dem theoretisch-philosophischen Ideal abgeglichen und beurteilt werden, beispielsweise in der Demokratiemessung (vgl. beispielsweise Bühlmann et al. 2012), oder (empirische) Legitimitätskriterien (normativ) in demokratische und nichtdemokratische Merkmale unterschieden werden, wie in der Studie Prekäre Legitimitäten (Nullmeier et al. 2010).

In diesem Sinne wird die terminologische Engführung auf zwei Ausrichtungen der Legitimitätsforschung schon länger beanstandet (vgl. beispielsweise Binder und Heupel 2015, S. 238, 240; Patberg 2013, S. 157; Zürn 2011, S. 606-607; Beetham und Lord 1998, S. 4-5). Thornhill (2011, S. 135, 137-138) kritisiert die Arbeitsteilung zwischen politischer Theorie und Philosophie und deren Zuständigkeit für die Elaborierung präskriptiver Normen auf der einen und der soziologischen Deskription von Einstellung oder Praktiken auf der anderen Seite und mahnt die Anerkennung des normativen Fundaments auf gesellschaftlicher Ebene an. Ähnlich plädiert Gaus (2011, S. 1, 4-7) dafür, einzubeziehen ,how ideas work in societal practice“ (Gaus 2011, S. 6) - in der Gesellschaft, der Öffentlichkeit, in politischen Institutionen. Tatsächlich wird diese Forderung bereits umgesetzt: durch einige der inhalts- und diskursanalytischen Studien der Legitimationsforschung ${ }^{1}$ (beispielsweise Nullmeier et al. 2010; Binder und Heupel 2015; Barnickel 2019) oder die - eher in der Demokratieforschung situierte - Erforschung unterschiedlicher Demokratieverständnisse (beispielsweise die Beiträge in Schubert und Weiß 2016a).

Bereits dieser kursorische Überblick verweist darauf, dass auch empirischer Legitimitätsforschung eine normative Dimension inhärent ist oder zumindest sein kann. Diese genauer zu situieren und die pluralen normativen Gehalte empirischer Legitimitätsforschung zu verorten, ist Ziel des vorliegenden Beitrags. Bei einem derart vielschichtigen Begriff sind die Grundlagen von Legitimitätsbewertung und -kritik $\mathrm{zu}$ bestimmen. Es ist also zu beachten, in wessen Urteil und auf Basis von wessen normativen Kriterien Legitimität attestiert wird (vgl. Barker 2007, S. 33).

Der Artikel geht hierzu wie folgt vor: Nach einer Annäherung an die Pluralität der empirischen Legitimationsforschung (Abschn. 2) wird argumentiert, dass eine Verknüpfung mit Erkenntnissen der Bewertungssoziologie sowie unterschiedlichen Kritikbegriffen instruktiv (vgl. Dammayr et al. 2015) ist, um systematisch zwischen Nuancen des Legitimitätsbegriffs zu unterscheiden (Abschn. 3). Denn beide nehmen Differenzierungen vor, die auch in der Legitimitätsforschung bestehen, jedoch durch den gemeinsamen begrifflichen Bezug auf Legitimität beziehungsweise letztlich die Beurteilung von Legitimität beizeiten verschwimmen. So wird illustriert, dass die normativen Gehalte auch in der empirischen Legitimitätsforschung komplexer gelagert sind als bestehende Unterscheidungen nahelegen. Diese Debattenstränge ver-

\footnotetext{
1 Der Begriff der Legitimation ist dynamischer angelegt als der statische Legitimitätsbegriff und betont die prozessuale Herstellung von Legitimität (vgl. Luckmann 1987, S. 110; Zürn 2012, S. 42). Für das hier verfolgte Argument ist diese - zweifelsohne wichtige - Unterscheidung nachrangig, daher werden die Begriffe unterschiedslos gebraucht.
} 
knüpfend wird eine Systematisierung entwickelt, um unterschiedliche Zugriffe (und auch Erkenntnisinteressen) auf Legitimität systematischer als bisher unterscheiden zu können (Abschn. 4). Diese Systematisierung folgt zwei Differenzierungen: Erstens wird zwischen Werten und Bewerten unterschieden sowie zweitens danach, wer jeweils wertet und bewertet. Den hieraus resultierenden Typen der Legitimationsforschung werden sodann exemplarisch Ansätze der bestehenden Forschung zugeordnet. Hierdurch wird nicht nur aufgezeigt, welch unterschiedliche Phänomene mit dem Legitimitätsbegriff gefasst werden und wie jeweils der normative Gehalt verschiedener Varianten der Legitimitätsforschung gelagert ist, sondern auch wie plural die Herangehensweisen, analytischen Fokusse sowie Gegenstände sind, die mit den Begriffen Legitimität und Legitimation bezeichnet werden. Dabei geht es dem Beitrag ausdrücklich nicht darum, für ein bestimmtes Modell der Legitimitätsforschung zu plädieren. Vielmehr wird durch die Auffächerung der Vielfalt des Umgangs mit der normativen Dimension - und damit auch der (sich ergänzenden) Varianten des Verständnisses von Legitimität - in der empirischen Forschung veranschaulicht, auf welchen Ebenen Kritik und Normativität jeweils verortet sind. Implikationen für eine (meta-)kritische Legitimitätsforschung werden abschließend diskutiert (Abschn. 5).

\section{Pluralität der empirischen Legitimitätsforschung: Eine Annäherung}

Dass in der Legitimitätsforschung eine Differenzierung zwischen gesellschaftlicher und wissenschaftlicher Legitimitätsbeurteilung vorzunehmen ist, ist nicht umstritten. So unterscheidet Dieter Fuchs (2011, S. 31) Einteilungen von Regimen als legitim oder illegitim auf der Basis bestimmter Anerkennungswürdigkeitskriterien durch Wissenschaftler*innen (bei Fuchs als „objektive Legitimität“ bezeichnet) von gesellschaftlichen Legitimitätsüberzeugungen (,,subjektive Legitimität“). Auf eine ähnliche Leitunterscheidung laufen die beiden ,Modelle empirischer Legitimitätsforschung“, so der Titel des Aufsatzes, bei Markus Patberg (2013) zu. Diese wichtige Differenzierung bei Patberg wird allerdings durch die Bezeichnung der Modelle als „Messmodell“2 und „Beurteilungsmodell““ überdeckt. Dennoch lässt sich anhand der Modelle instruktiv verdeutlichen, dass nicht nur die Legitimitätsbeurteilung auf wissenschaftlicher oder gesellschaftlicher Ebene situiert sein kann, sondern diese Unterscheidung auch für die Provenienz der normativen Grundlage dieser Beurteilung zutrifft.

Unter das Messmodell fasst Patberg die klassische Variante der Einstellungsforschung, die Akzeptanz, Unterstützung oder Vertrauen in einem Survey-Design misst. Obwohl durch Patberg dem Beurteilungsmodell entgegengesetzt, liegt somit auch im Messmodell eine Beurteilungsdimension vor: Die Legitimitätsbeurteilung liegt bei den befragten Bürger*innen, deren Urteile - operationalisiert als Unterstützung

\footnotetext{
${ }^{2}$ Die Wahl des Begriffs „Messmodell“ bei Patberg ist auch deshalb irreführend, da mit „Messmodell“ meist die Operationalisierung latenter in manifeste Variablen bezeichnet wird. In diesem Sinn gebraucht Patberg den Begriff allerdings nicht. Diesen Hinweis verdanke ich einem*einer der anonymen Gutachter*innen.
} 
oder Vertrauen - die Forschung ermittelt (vgl. Patberg 2013, S. 157-158). Dieses Modell kritisiert Patberg für den fehlenden Einbezug der „Gründe für die Zuschreibung oder Nicht-Zuschreibung von Legitimität“" (Patberg 2013, S. 158) durch die Bürger*innen - mithin die fehlende Normativität.

Normativität spielt für Patberg in empirischer Legitimitätsforschung im Sinne des Beurteilungsmodells eine Rolle. Hierunter fasst er Legitimitätsforschung, die die ,normativen Erwartungen der Bürger“ identifiziert, um diese mit den durch die Forscher*innen rekonstruierten „Strukturen und der Performanz des politischen Systems“" (beide Zitate bei Patberg 2013, S. 160) abzugleichen (ähnlich Zürn 2011, S. 606). Die Legitimitätsbeurteilung wandert hier auf die Ebene der Forscher*innen, welchen es obliegt, die Übereinstimmung normativer Erwartungen und rekonstruierter Herrschaftsstrukturen zu beurteilen. Der durch die Forscher*innen attestierte Grad der Übereinstimmung indiziert dann den Grad der empirischen Legitimität (vgl. Patberg 2013, S. 160).

Gegenüber normativer Legitimitätsforschung unterscheidet sich das Beurteilungsmodell hinsichtlich der Ebene, auf der der normative Beurteilungsmaßstab konstruiert wird. Hier wird dieser nicht aus der politischen Theorie entwickelt, sondern ergibt sich aus den Erwartungen der Bürger*innen (vgl. Patberg 2013, S. 159, 163). Dieser Umstand verweist auf die zweite Unterscheidungslinie neben jener der Ebene der Legitimitätsbeurteilung: Nicht nur die Legitimitätsevaluation, sondern auch die Normkonstruktion können sowohl gesellschaftlich als auch wissenschaftlichtheoretisch inspiriert sein.

Somit verengt die Reduktion auf lediglich zwei Modelle die analytischen $\mathrm{Zu}$ griffe auf empirische Legitimität. Es sind unter anderem solche Perspektiven der Legitimitätsforschung weder dem Mess- noch dem Beurteilungsmodell zuzuordnen, die sowohl die Legitimitätsurteile als auch die normativen Maßstäbe auf gesellschaftlicher Ebene untersuchen. So ist durchaus denkbar, dass Bürger*innen die Übereinstimmung zwischen Herrschaftsordnung und normativen Erwartungen anders wahrnehmen als die Forschenden (im Beurteilungsmodell) es tun. ${ }^{3}$

Will man Varianten der empirischen Legitimitätsforschung systematisierend einordnen, ist dieser doppelten Unterscheidungslinie Rechnung zu tragen: Es ist zu

\footnotetext{
${ }^{3}$ Diese Möglichkeit ist Patberg zwar bewusst, sie bleibt aber ohne Auswirkungen auf die Modelle (vgl. Patberg 2013, S. 168-169). Patberg exkludiert aus seiner Konzeption zwar normative Ansätze und trifft die Zuordnung danach, „ob der Maßstab, der dem Legitimitätsurteil zugrunde gelegt wird, aus der Empirie oder aus der normativen Theorie gewonnen wird“ (Patberg 2013, S. 163, Hervorhebung dort), also ob er induktiv oder deduktiv erlangt wird. Diese Konzeption ist aus zwei Gründen zu reduziert. Erstens geht es im Messmodell gerade nicht um die Erhebung von Maßstäben, sondern ausschließlich um die Messung von Legitimitätsurteilen. Hier ist das Zuordnungskriterium zu empirischer gegenüber normativer Forschung nicht gegeben. Zweitens ist nicht offenkundig, warum empirische Forschung (im Beurteilungsmodell) nicht Maßstäbe aus der Theorie deduzieren sollte, deren empirische Geltung dann rekonstruiert wird. Dies tun die Autor*innen in Nullmeier et al. (2010, 2014), deren Ansatz nur schwerlich als normativ-theoretische Debatte durchgehen wird. Induktive Forschung ist also genauso wenig zwangsläufig mit einem Interesse für Beurteilungsmaßstäbe der Herrschaftsunterworfenen gleichzusetzen, wie es deduktive Vorgehensweisen mit Maßstäben der politischen Theorie sind.
} 
differenzieren, wessen normativer Maßstab angelegt wird und wer das Legitimitätsurteil fällt. ${ }^{4}$

\section{Werten und Bewerten und ihr Verhältnis zu Normativität und Kritik}

Diese doppelte Unterscheidungslinie lässt sich instruktiv mit der bewertungssoziologischen Debatte um Werten und Bewerten sowie mit Konzeptionen von Normativität und Kritik verknüpfen. So kann der normative Standpunkt von (Legitimitäts-)Kritik konziser gefasst und unterschiedliche Zugriffe auf Legitimität unterschieden werden. Dies ist - trotz des vielleicht etwas komplex wirkenden Begriffsapparats instruktiv: zum einen, um Varianten von Normativität und Kritik in der empirischen Legitimationsforschung genauer zu situieren, und zum anderen, weil die Nähe zu unterschiedlichen Kritikbegriffen verschiedene wissenschaftliche Selbstverständnisse offenlegt.

Die Unterscheidung zwischen (normativem) Werten und (evaluativem) Bewerten - die sich durch die englischen Begrifflichkeiten valuation und evaluation vielleicht etwas prägnanter fassen lässt - hebt darauf $a b$, dass Werten eine Vorbedingung für Bewerten darstellt. In Praktiken des Wertens werden zunächst Sinn- oder Bedeutungsordnungen geschaffen, anhand derer Objekte erst bewertet oder beurteilt, also die Erfüllung der Werte überprüft, werden können (vgl. Krüger und Reinhart 2016, S. 492-493; Vatin 2013, S. 31; Lamont 2012, S. 205).

Praktiken des Bewertens und des Beurteilens stehen in Verbindung zu Kritik. So ,[stellt sich] [d]ie Frage nach den Bedingungen und der Möglichkeit von Kritik [...] immer dort, wo Gegebenheiten analysiert, beurteilt oder als falsch abgelehnt werden“ (Jaeggi und Wesche 2009, S. 7). Etymologisch stammt der Begriff vom griechischen Adjektiv kritikós beziehungsweise dem Verb krínein her, welches auf die Unterscheidungs- und Urteilsfähigkeit verweist (vgl. Röttgers 1982, S. 651; Bittner 2009, S. 135). Obwohl der Begriff in der historischen Genese einiger Veränderung unterlag, beispielsweise hinsichtlich dessen, worauf sich Kritik richtet und wer mit welcher Legitimation als Kritiker*in auftritt oder auftreten kann (vgl. ausführlich Röttgers 1982; Vobruba 2013, S. 148-153), ist der Bezug zur Beurteilung erhalten geblieben (vgl. Bittner 2009, S. 137).

Das verweist darauf, dass, wenn wir Kritik - zunächst noch etwas verkürzend als den Akt oder auch Prozess des Beurteilens oder Bewertens ansehen, Kritik auf unterschiedlichen Ebenen angesiedelt sein kann. Dies steht in Bezug zur Nähe oder Distanz der Urteilenden (vgl. Jaeggi und Wesche 2009, S. 9): Sind es gesellschaftliche Akteur*innen, deren Urteil über soziopolitische Situationen im Fokus steht, oder sind es Wissenschaftler*innen, die aus einer (vermeintlich) distanzierten Beobach-

\footnotetext{
4 Ähnlich argumentiert Fuchs (2011, S. 29-31), dass unterschieden werden müsse, welche Normen als Evaluationsbasis gelten sollen und wer schließlich die Beurteilung vornimmt. Allerdings bezieht Fuchs die Frage nach der Angemessenheit von Normen vor allem auf die wissenschaftlich-theoretische Debatte, weniger auf die gesellschaftlich als angemessen anerkannten Normen. Somit findet auch hier eine allerdings anders als bei Patberg gelagerte - Engführung statt.
} 
ter*innenposition den identischen Gegenstand kritisieren? Dem liegen verschiedene Verständnisse dessen zugrunde,

ob eine sich als kritisch verstehende Sozialforschung in der Lage ist, selbst Kritik zu äußern und von welcher Position aus sie dies gegebenenfalls kann, oder ob sie sich darauf beschränken muss, die von den AkteurInnen geäußerte Kritik [...] wiederzugeben (Dammayr et al. 2015, S. 13).

Sowohl für die Position, Aufgabe der Wissenschaft sei zuvorderst die Rekonstruktion der gesellschaftlichen Kritik(en), als auch für die Auffassung, wissenschaftliche Kritik erschöpfe sich eben nicht in dem rekonstruktiven Moment, sondern habe eine eigenständige kritische Perspektive, finden sich Vertreter*innen.

Für die erste Position kann exemplarisch der Kritikbegriff bei Luc Boltanski stehen (vgl. Vobruba 2013, S. 161-162; Dammayr et al. 2015, S. 13), dem es primär um das Herausarbeiten der kritischen Fähigkeiten der gesellschaftlichen Akteur*innen geht (vgl. Boltanski 2010, S. 48). Dieser „Soziologie der Kritik“ steht mit der „Kritischen Soziologie“ (Boltanski und Honneth 2009) eine zweite Perspektive entgegen (vgl. Vobruba 2013, S. 155-156, 161-162). Hier ist das kritische Moment anders gelagert: Aufgabe der Wissenschaft ist nicht die Rekonstruktion der in der Gesellschaft präsenten Kritik, sondern die Bewertung durch die Wissenschaft selbst (vgl. Bittner 2009, S. 139-140, 143). Dies kann - wie bei Horkheimer - mit einer emanzipatorischen Perspektive verknüpft sein (vgl. Horkheimer und Marcuse 1937, S. $\left.626^{5}\right)$.

So wie die Bewertungssoziologie zwischen Praktiken des Bewertens und Wertens unterscheidet, so ist Kritik nicht auf den beurteilenden (bewertenden) Akt zu begrenzen (vgl. Allerkamp et al. 2015, S. 11). Vielmehr baut sie auf „Wissensformen“ (Allerkamp et al. 2015, S. 12), auf Wertungsordnungen, auf, die ein kritisches Urteil erst ermöglichen. Das Urteil selbst steht also in Verbindung zu normativen Wertungspositionen und ist auf seine normativen Grundlagen hin zu befragen.

Bei Boltanski und Thévenot ist die Normativität in gesellschaftlichen Maßstäben (vgl. Boltanski und Thévenot 2014, S. 61), bezeichnet als „Rechtfertigungsordnung[en]" (Boltanski und Thévenot 2014, S. 108), situiert. Auf deren Basis wird Kritik - in Form von „Prüfungen“ (Boltanski 2010, S. 47, 155, 172) - formuliert. Eine solchermaßen normativ verankerte Kritik wird bisweilen als ,schwache Normativität" (O’Neill 2000, S. 719) konzipiert, welche den Rechtfertigungsmaßstab entlang seiner Geltung in einer sozialen Ordnung bestimmt. Demgegenüber stehen solche Ansätze, die eine „starke Normativität“ (O’Neill 2000, S. 719) verfolgen und ihren Rechtfertigungsmaßstab aus der Annahme und Begründung universell gültiger Normen gewinnen (vgl. O’Neill 2000, S. 719-720; zur Unterscheidung von starker und schwacher Normativität beziehungsweise immanenter und transzendenter Kritik vgl. auch Jaeggi und Wesche 2009, S. 8-9; Wesche 2009, S. 203-220). Dies verweist auf den je verschiedenen Horizont des Wertens, also des Ideals, das definiert, wie eine bestimmte Situation oder Praxis beschaffen sein soll (vgl. Vobruba 2013, S. 153-154; Baran und Wagner 1990, S. 574).

\footnotetext{
5 Horkheimer und Marcuse zeichnen in dem Text für getrennte Abschnitte verantwortlich. Der zitierte Ausschnitt stammt aus dem Beitrag Max Horkheimers.
} 


\section{Varianten von Normativität und Kritik in der empirischen Legitimationsforschung}

Die Unterscheidung zwischen Werten und Bewerten und dabei insbesondere die Differenzierung unterschiedlicher Ebenen, wie sie in den Debatten zu Normativität und Kritik verhandelt wird, spiegelt also die Differenzierungslinien, die in der Legitimationsforschung bestehen. Greift man diese auf, ergeben sich neun mögliche Varianten der Legitimationsforschung, wie Tab. 1 darstellt. Sie trägt in den Zeilen $\mathrm{ab}$, wessen normativer Bewertungsmaßstab angelegt wird (normativ-wertende Dimension). Die Spalten bezeichnen, durch wen die Bewertung erfolgt, wer also das Legitimitätsurteil fällt (kritisch-bewertende/beurteilende Dimension).

Der normative Maßstab ist mit einer spezifischen „Wertungsposition“, das Legitimitätsurteil mit einer spezifischen kritischen „Bewertungsposition“ verbunden. Zudem sind beide Dimensionen verknüpft: Die kritische Bewertung, also das Legitimitätsurteil in den Spalten der Tab. 1, ist nur möglich, wenn zuvor ein Maßstab des Wertens - beispielsweise bestimmte Sollens-Normen - etabliert ist (vgl. Krüger und Reinhart 2016, S. 492-493), also gesellschaftlich formierte (Legitimitäts-)Normen oder philosophisch hergeleitete (Legitimitäts-)Ideale (vgl. Jaeggi und Wesche 2009, S. 7-9), wie sie die Zeilen der Tabelle abtragen.

In alltäglichen Prozessen sind Werten und Bewerten verwoben (vgl. Vatin 2013, S. 45; Lamont 2012, S. 205). Analytisch und forschungspraktisch ist aber durchaus trennbar, wessen Wertungsmaßstab herangezogen wird und wessen Urteil letztlich in die Betrachtung einfließt. In den Zellen der Tabelle ergeben sich dann unterschiedliche Rekombinationen der normativ-wertenden und kritisch-bewertenden Dimension. Die Nummerierung ist nicht als Sequenz oder Hierarchisierung zu lesen, sondern dient lediglich der Kennzeichnung.

Sowohl der normative Maßstab (in den Zeilen) als auch das Legitimitätsurteil (in den Spalten) kann entweder ,extern“, aus der wissenschaftlichen Beobachter*innenposition, formuliert sein oder Forschung kann sich darauf richten, ,interne" normative Maßstäbe und Urteile, also beispielsweise der Bürger*innen oder der politischen Eliten, zu rekonstruieren. Darüber hinaus kann in der konkreten Forschungsanlage auf die Erhebung oder Formulierung einer der beiden Dimensionen verzichtet werden (jeweils in der ersten Zeile und Spalte der Tabelle verortet). So können beispielsweise Legitimitätsurteile der Bevölkerung erforscht werden, die zu Grunde liegenden Maßstäbe aber nicht. Über die Zuordnung der Ansätze entschei-

Tab. 1 Varianten der Legitimitätsforschung ${ }^{\mathrm{a}}$

\begin{tabular}{|c|c|c|c|}
\hline $\begin{array}{l}\text { Bewerten/kritisches } \\
\text { Legitimitätsurteil }\end{array}$ & $\varnothing$ & $\begin{array}{l}\text { Intern/gesellschaft- } \\
\text { liche Ebene }\end{array}$ & $\begin{array}{l}\text { Extern/wissenschaftlich- } \\
\text { theoretische Ebene }\end{array}$ \\
\hline \multicolumn{4}{|l|}{$\begin{array}{l}\text { Werten/normativer } \\
\text { Legitimitätsmaßstab }\end{array}$} \\
\hline$\varnothing$ & I & II & III \\
\hline Intern/gesellschaftliche Ebene & IV & $\mathrm{V}$ & VI \\
\hline Extern/wissenschaftliche Ebene & VII & VIII & IX \\
\hline
\end{tabular}

Quelle: Eigene Darstellung

a Die dargestellten Varianten führen die Überlegungen in Barnickel (2019, S. 28) substanziell weiter 
det, welche Dimensionen in welcher Kombination jeweils analytisch einbezogen werden. Dies bedeutet keineswegs, dass der beforschte Gegenstand nicht auch hinsichtlich der zweiten Unterscheidungslinie betrachtet werden könnte, in dem genannten Beispiel also nicht nur die Legitimitätsurteile der Bevölkerung analysiert werden können, sondern auch die gesellschaftlichen normativen Maßstäbe, auf denen die Urteile fußen. Gleichwohl gibt es viele Beispiele der Legitimitätsforschung, die sich auf eine der Unterscheidungslinien fokussieren und die andere ausblenden. ${ }^{6}$

Immer rückt der jeweilige analytische Zuschnitt eine spezifische Kombination in den Fokus und spart andere aus. Folglich repräsentieren unterschiedliche Ansätze der Legitimationsforschung je spezifische Rekombinationen der wertenden und der bewertenden Ebene und damit auch verschiedene Varianten von Normativität und Kritik. Daher stellen die Felder der Tabelle nicht alternative - und letztlich austauschbare - Forschungsanlagen dar. Vielmehr heben sie auf unterschiedliche Fragen und Aspekte von (empirischer) Legitimität ab, die sich ergänzen können aber nicht substituieren.

Im Folgenden werden die Zellen der Tabelle - in der Reihenfolge der Zeilen - erläutert und Ansätze aus der Legitimations- (und der Demokratie-)forschung ${ }^{7}$ beispielhaft zugeordnet. Dabei sind zwar alle Kombinationen, wie sie die Felder der Tabelle abbilden, theoretisch denkbar. Sie werden aber forschungspraktisch nicht gleichermaßen stark bedient - teilweise aus guten Gründen, beispielsweise wenn Kriterien der wissenschaftlichen Redlichkeit nur bedingt Genüge getan würde.

In der nun folgenden Diskussion der Felder der Tabelle muss die Auswahl der zugeordneten Ansätze notwendig selektiv bleiben und erhebt nicht den Anspruch, eine erschöpfende Darstellung der Legitimitätsforschung in ihrer gesamten Breite zu leisten. Vielmehr dient sie der Illustration der jeweiligen Verknüpfung der normativen und der kritischen Dimension und damit der Verschiedenheit des normativen Gehalts auch in der empirischen Legitimitätsforschung. Zudem reduziert die Darstellung in Zellen die Komplexität der Ansätze. Auch innerhalb der Zellen können - wie im Folgenden deutlich wird - Unterschiede bestehen, z. B. hinsichtlich der Substanz des normativen Maßstabs, der Methodologie oder danach, wie ambitioniert ein Wertungsmaßstab expliziert wird und/oder ob Schwerpunkte auf die wertende oder die bewertende Dimension gesetzt werden.

\subsection{Kein normativer Legitimitätsmaßstab, verschiedene Ebenen der Legitimitätsbeurteilung}

Gemeinsam ist den Ansätzen der Felder I bis III, dass kein normativer Maßstab vorliegt beziehungsweise der analytische Fokus die normativen Maßstäbe (also die Wertungsdimension), auf denen ein Legitimitätsurteil fußt, ausspart. Sie unterschei-

\footnotetext{
6 Wie noch näher ausgeführt wird, ist mit der Möglichkeit der Fokussierung auf eine der Dimensionen nicht negiert, dass sich Kritik nicht im Beurteilungsakt erschöpft, sondern auf einem normativen Rahmen basiert.

7 Varianten der Demokratieforschung sind hier insoweit eingezogen, als sie (implizit oder explizit) Bezüge zur Legitimitätsforschung aufweisen.
} 
den sich dahingehend, ob beziehungsweise durch wen eine Bewertung vorgenommen wird, also das Legitimitätsurteil getroffen wird.

\subsubsection{Feld I: kein Werten, kein Bewerten}

Der erste Typ von Ansätzen der Legitimationsforschung kümmert sich nicht nur nicht um die Etablierung oder Rekonstruktion eines normativen Maßstabs, sondern es geht solchen Ansätzen auch nicht um die Bewertung konkreter Legitimationsobjekte. Hier steht also weder der normative Maßstab, noch das Fällen eines Legitimitätsurteils im Forschungsinteresse. Am ehesten ist eine solche Kombination wohl in der empirischen Legitimationstheorie zu finden. Niklas Luhmann (2008[1969]) beispielsweise argumentiert anhand der Deskription von Verfahren, dass die Funktion solcher Verfahren in der Legitimation liegt. Legitimität meint dann die ,generalisierte Bereitschaft, inhaltlich noch unbestimmte Entscheidungen innerhalb gewisser Toleranzgrenzen hinzunehmen“ (Luhmann 2008[1969], S. 28, Hervorhebung dort), die (normativen) Grundlagen dieser Bereitschaft und das Ausmaß der tatsächlich bestehenden Bereitschaft bleiben offen. ${ }^{8}$ Insbesondere liegt die Absicht nicht darin, präskriptiv Normen zu begründen, deren Erfüllung über die Anerkennungswürdigkeit einer Ordnung entscheidet. Vielmehr geht es hier um eine funktionale Perspektive auf Verfahren, welche als Eigenleistung des Systems durch Selektionsprozesse Komplexität reduzieren und dadurch Legitimation - und über die Kleinarbeitung von Widerstand letztlich Stabilität - herstellen (vgl. Luhmann 2008[1969], S. 4, 25, 30).

\subsubsection{Feld II: kein Werten, internes Bewerten}

Feld II umfasst solche Ansätze, die ebenfalls keinen bestimmten normativen Standard anlegen oder explizieren, zugleich aber auf die Erhebung der intern-gesellschaftlichen Bewertung von Legitimität ausgerichtet sind. Auch hier findet sich mit den Arbeiten Max Webers ein Ansatzpunkt in der Legitimitätstheorie. Diesem geht es, wie oben ausgeführt, um die Orientierung von Handeln an der Ordnung, ohne ein besonderes Interesse für die normativen Grundlagen der Orientierung. ${ }^{9}$ Ähnlich betont Seymour M. Lipset den Glauben an Legitimität, wenn er diese definiert als

the capacity of the system to engender and maintain the belief that the existing institutions are the most appropriate ones for the society (Lipset 1983[1959],

S. 64).

\footnotetext{
${ }^{8}$ Zwar wird diskutiert, inwieweit nicht auch bei Luhmann eine minimale normative Dimension inhärent ist, sind doch die Toleranzgrenzen vermutlich normativ umrissen (vgl. Gaus 2011, S. 11; Lübbe 1991, S. 128, 131-135). Entscheidend für die Zuordnung hier ist aber, dass der Gehalt dieser Normen für die Argumentation bei Luhmann keine Rolle spielt.

9 Damit ist nicht negiert, dass Weber mit seiner Unterscheidung der traditionalen, der charismatischen und der legal-rationalen Herrschaft unterschiedliche Grundlagen der drei Herrschaftstypen anlegt, so dass auch hier eine motivhafte Orientierung an der Ordnung angenommen wird. Dennoch richtet sich das vorrangige Interesse - und so hat es die Rezeption aufgenommen - nicht auf die normative Substanz, sondern auf die Folgebereitschaft beziehungsweise den Legitimitätsglauben (vgl. Weber 1980[1921], S. 17-20; Gaus 2011, S. 9).
} 
David Eastons (1965, S. 157, 165) Arbeiten, die weite Teile der empirischen Legitimitätsforschung inspiriert haben, heben auf das Ausmaß an (spezifischer und vor allem) diffuser Unterstützung ab, die sich jeweils auf unterschiedliche Legitimationsobjekte (Gemeinschaft, Regime, Autoritäten) richtet. Hinsichtlich der Legitimitätsfrage geht es im Kern darum, wie Bürger*innen bestimmte Objekte beurteilen. Sowohl Easton (1965, S. 158, 161) als auch Lipset (1983[1959], S. 68) verbinden die Legitimitätsfrage funktional mit der Bedeutung von Legitimität für Systemstabilität. Ohne die gängige Einordnung als funktionale Perspektive bestreiten zu wollen, erhellt die hier vorgenommene Differenzierung nun, dass gleichwohl mit dem Fokus auf die gesellschaftliche Legitimitätsbeurteilung auch eine kritische Perspektive, verstanden als gesellschaftliche Bewertung, verbunden ist.

Obwohl die genannten Autor*innen in unterschiedlichem Maße auch auf die normative Basis dieser Legitimitätsbeurteilung hinweisen, wird in der Rezeption und der forschungspraktischen Umsetzung häufig vorrangig auf die Erhebung der Legitimitätsbeurteilung abgestellt. ${ }^{10}$ Besonders die Einstellungsforschung hat die genannten Impulse aufgenommen und ist dann hier zu subsumieren, wenn es um die Akzeptanz und Unterstützung der Bürger*innen gegenüber der politischen Ordnung, ihren Institutionen oder Autoritäten geht (vgl. beispielsweise Gilley 2012). Arbeiten in dieser Forschungsanlage entsprechen also einem „Messmodell“", wie Patberg (2013) es beschreibt. Erfragt wird dann die Demokratiezufriedenheit oder das Vertrauen in Institutionen (vgl. beispielsweise Westle 2007). Viele dieser Ansätze diskutieren die Ursachen (als unabhängige Variablen) für die gemessene empirische Legitimität. So durchleuchten Dellmuth und Tallberg (2015) die empirische Legitimität (gemessen über Vertrauen [vgl. Dellmuth und Tallberg 2015, S. 461, 475]) der Vereinten Nationen dahingehend, inwieweit die Legitimität von Interessenrepräsentation, Performanz und Vertrauen in andere Institutionen abhängt. Aarts et al. (2017) untersuchen den Zusammenhang von Demokratiezufriedenheit und (post-)materialistischen Wertorientierungen.

Legitimitätsurteile werden nicht nur über Einstellungen erhoben, sondern auch in der Verhaltensforschung, die aus beobachtbarem Verhalten der (non-)compliance (wie Protestverhalten, Wahlbeteiligung) auf die Beurteilung der Legitimität durch die Bürger*innen schließt. Allerdings ist der Schluss von beobachtetem Verhalten auf Legitimität unsicherheitsbehaftet, worauf bereits Easton (1965, S. 159-160) in der Diskussion seines Konzepts des „overt support“ hingewiesen hat. So kann Protest ein Hinweis auf die Delegitimation einer Ordnung sein (mithin ein negatives Legitimitätsurteil) oder Ausweis der (demokratischen) Legitimität, wenn kritische Bürger*innen (vgl. Norris 1999) sich beteiligen (zu diesem Argument vgl. ausführlicher Schmidtke und Schneider 2012, S. 233-235; Gaus 2011, S. 8).

Ein linguistisch ausgerichteter Strang der Legitimationsforschung interessiert sich dafür, auf welche diskursiven Strategien (insbesondere politische) Akteur*innen zurückgreifen, um politische Entscheidungen oder Institutionen als legitim (oder illegitim) zu konstruieren. Diese Arbeiten rekonstruieren häufig die bei van Leeuwen

10 Ansätze, die neben der Legitimitätsbeurteilung auch die normativen Erwartungen der Bürger*innen erheben, sind hingegen Feld V zuzuordnen, selbst wenn sie ein ähnliches methodisches Instrumentarium verwenden und sich auf identische Referenzautor*innen stützen. 
und Wodak (1999) und van Leeuwen (2007) herausgearbeiteten Strategien: Bezug auf Autoritäten, Rationalisierung, Moralisierung und Storytelling (für Anwendungen und Weiterentwicklungen vgl. beispielsweise Rojo und van Dijk 1997; Vaara 2014). Sofern hier nicht der normative Referenzrahmen der Sprecher*innen Teil des analytischen Fokus ist, sind solche Diskursanalysen unter Feld II zu subsumieren.

Trotz der deutlich verschiedenen methodologischen Zugriffe eint diese Ansätze der Umgang mit der Leitunterscheidung: Sie interessieren sich für den kritisch bewertenden Aspekt, welcher auf gesellschaftlicher Ebene verortet und gemessen oder rekonstruiert wird, nicht aber für den normativ-wertenden Aspekt von Kritik, da auf die Erhebung der normativen Grundierung der Kritik verzichtet wird.

\subsubsection{Feld III: kein Werten, externes Bewerten}

Feld III beschreibt Ansätze, die ein wissenschaftliches (Legitimitäts-)Urteil fällen, auf eine Explizierung der normativen Grundlagen, auf denen das Urteil fußt, allerdings verzichten. Dieses Feld sollte leer bleiben, denn ein wissenschaftliches Urteil, dessen Prämissen und theoretischer Rahmen im Verborgenen bleibt, sollte nicht existieren. Gerring und Yesnowitz (2006, S. 106-108) sehen allerdings solche Urteile durch die Trennung von empirischer und normativ-philosophischer Wissenschaft befördert, gerade wenn sich in deskriptiv-analytisch angelegte Studien implizite normative Urteile einschleichen.

\subsection{Wissenschaftlich-theoretischer Legitimitätsmaßstab, verschiedene Ebenen der Legitimitätsbeurteilung}

Während die Ansätze in den Feldern I bis III keine normativen Maßstäbe analytisch in Anschlag bringen, beziehen die Ansätze in der untersten Zeile der Tabelle diese extern aus der wissenschaftlich-theoretischen Debatte. Abermals unterscheiden sie sich dahingehend, wer auf dieser Basis die Legitimität bewertet. Die Bewertung kann wieder auf wissenschaftlicher oder gesellschaftlicher Ebene vorgenommen werden oder gänzlich fehlen - dann nämlich, wenn kein Legitimitätsurteil gefällt wird. Die Erläuterung der Felder der letzten Zeile der Tabelle wird vorgezogen, da hier einige „klassische“ Ansätze der Legitimitätsforschung verortet sind, wohingegen die mittlere Zeile wohl die meisten innovativen Zugriffe vereint, sodass diese abschließend diskutiert werden.

\subsubsection{Feld VII: externes Werten, kein Bewerten}

Die Kombination externes Werten/kein Bewerten formuliert ein normatives Ideal, nimmt allerdings keine Legitimitätsbewertung vor. Solche Ansätze richten sich durch den Verzicht auf den Bewertungsakt nicht auf einen konkreten Gegenstand, dessen Legitimität beurteilt werden soll. Sie sind vorrangig in der politischen Theorie zu finden und somit eher der normativen, nicht der empirischen, Legitimitätsforschung zuzuordnen. Dennoch sei knapp auf dieses Feld eingegangen, nicht zuletzt da Arbeiten in den Feldern VIII und IX die Ansätze in Feld VII zur normativen Bewertungsfolie machen (können). Gemeinsam ist den Theorien innerhalb dieses Feldes 
die Etablierung normativer Kriterien für eine $\mathrm{Zu}$ - oder Absprechung von Legitimität, häufig verstanden als demokratische Legitimität (vgl. Peter 2009, S. 57).

Sowohl die Kriterien selbst als auch der Fokus auf den Ort der Legitimitätsgenerierung im politischen Prozess - im Sinne der Input-, Throughput- und OutputLegitimität (vgl. Scharpf 1999, S. 16-28; Schmidt 2013) - offenbart die Pluralität der Ansätze. So unterscheidet beispielsweise Fabienne Peter (2009, S. 2-3, 62-63) prozedurale Ansätze und solche, die Prozess und Qualität der Ergebnisse in den Fokus nehmen von solchen, die Legitimität von der Erreichung politischer Ergebnisse (wie Gleichheit) abhängig machen.

So machen deliberative Ansätze ,den Verfahrensbegriff der deliberativen Politik zum normativ gehaltvollen Kernstück der Demokratietheorie“ (Habermas 1996, S. 285), bei einigen Strömungen auch gekoppelt mit der Annahme des Beitrags zur Erzielung ,vernünftige[r] bzw. faire[r] Ergebnisse“ (Habermas 1996, S. 286). Joshua Cohen definiert eine ,ideal deliberative procedure“ (Cohen 1989, S. 22), die unter anderem auf Freiheit und Gleichheit des Deliberationsprozesses setzt. Dieselben Werte werden im liberalen - mit der Betonung negativer Freiheiten - und republikanischen Demokratiemodell - mit dem Fokus auf positive Rechte und Gleichheit - betont, sind aber nicht als deliberativ-prozedurale Normen gefasst (vgl. Lembcke et al. 2012b, S. 17, 19; zur Unterscheidung von liberalem, demokratischem und deliberativem Demokratiemodell vgl. Habermas 1996, S. 277-292).

Die Perspektiven sind also plural - und häufig unvereinbar. Peter (2009, S. 2) attestiert eine ,increasing diversity of conceptions of democratic legitimacy“ und plädiert selbst für einen „Pure Epistemic Proceduralism“ (Peter 2009, S. 132). Auch normative Demokratietheorien, die letztlich häufig die Kriterien demokratischer Legitimität setzen, weisen eine ,Vielfalt der normativen Positionen auf“ (Lembcke et al. 2012b, S. 11) und explizieren (jeweils verschiedene) demokratische Idealmodelle (vgl. für einen Überblick die Beiträge in Lembcke et al. 2012a; sowie die Übersicht bei Lembcke et al. 2012b, S. 24). Hinzu kommen Ansätze, die die (theoretische) Definition des normativen Maßstabs nicht demokratietheoretisch fundieren, wie es in Konzepten der Good Governance der Fall ist (vgl. beispielsweise Bürgler 2009).

Für das vorliegende Argument ist - ohne die deutlichen Unterschiede negieren zu wollen - bedeutsam, dass jeweils eine wissenschaftlich-theoretische Normdefinition als Kriterium der „Anerkennungswürdigkeit“ (Habermas 1976, S. 39) stattfindet sei sie nun substanziell oder prozedural gewendet.

\subsubsection{Feld VIII: externes Werten, internes Bewerten}

Die Kombination des extern-wissenschaftlichen Wertens und einer internen Bewertung scheint zwar theoretisch möglich, aber praktisch schwer denkbar. Dies würde implizieren, einen normativen Bewertungsmaßstab extern vorzugeben, welchen Bürger*innen dann zur Grundlage ihrer Legitimitätsurteile machen müssten. Eine solche Kombination wäre exakt spiegelbildlich zu Patbergs Beurteilungsmodell. Mit der Annahme der Nichtexistenz dieser Kombination der Legitimationsforschung ist natürlich nicht negiert, dass wissenschaftliche normative Maßstäbe gesellschaftliche Relevanz besitzen und Diskurse prägen können. Sie allerdings als geschlossenen Be- 
wertungsmaßstab zu unterstellen und gleichsam anzunehmen, Bürger*innen könnten (und sollten) ihre Legitimitätsurteile ausschließlich auf extern vorgegebene Kriterien stützen scheint nicht plausibel. ${ }^{11}$

\subsubsection{Feld IX: externes Werten, externes Bewerten}

Feld IX bezeichnet Ansätze, die vor einem wissenschaftlich-theoretischen normativen Maßstab die Legitimitätsbeurteilung auch extern, also durch Forscher*innen, vornehmen. Dies sind beispielsweise Verfahren, die Demokratiequalität messen und letztlich abschätzen, inwieweit durch die Institutionalisierung von Demokratie Herrschaftsausübung legitim ist (vgl. für eine Übersicht und Diskussion Pickel und Pickel 2012; Campbell und Barth 2009; Bein 2018). Zwar stehen sich hier mit FreedomHouse ${ }^{12}$, Polity $I V^{13}$, dem Demokratiebarometer (Bühlmann et al. 2012) oder der Demokratiematrix ${ }^{14}$ Indizes gegenüber, die Demokratie (häufig angelehnt an liberale Demokratiemodelle) unterschiedlich operationalisieren und messen (vgl. für einen Vorschlag zur Messung radikaler Demokratiekonzeptionen Dlabac und Schaub 2012). Die normativen Maßstäbe können so plural sein wie die Theorien in Feld VII, dienen diese doch häufig als normative Folie. Gemeinsam ist ihnen nichtsdestoweniger, dass - und daher ist es für das Argument hier unerheblich, mit welchem Demokratiebegriff gearbeitet wird - die Definition dessen, was als demokratisch zu konzeptualisieren ist, aus einer theoretischen Reflexion entsteht. Diese bildet dann den Hintergrund für die Bewertung der Legitimität der Ordnung, verstanden als das Ausmaß ihrer Demokratiehaftigkeit.

Auch fallen viele der Debattenbeiträge zum Demokratie- und/oder Legitimitätsdefizit der Europäischen Union unter dieses Verständnis (für Überblicke vgl. beispielsweise Eriksen und Fossum 2004; Føllesdal 2006). Anführen ließe sich die Debatte um das Vorhandensein einer kollektiven Identität europäischer Bürger*innen als Voraussetzung (demokratischer) Legitimität - verbunden mit, je nach Definition des Maßstabs, skeptischen oder optimistischen Legitimitätsurteilen (vgl. beispielsweise Fuchs 2000; Kielmansegg 2003, S. 57-58).

Die Kontrastierung von Demokratiemessung und den Studien zur Europäischen Union zeigt bereits unterschiedliche Schwerpunktsetzungen der Zugriffe. Während das Legitimitätsurteil bei der Demokratiemessung häufiger implizit erfolgt, ist es in

\footnotetext{
11 Abzugrenzen sind (mindestens) zwei Ansätze, die vorschnell eingeordnet werden könnten. Dies sind, erstens, Ansätze, in denen Akteur*innen Evaluationen nach vorgegebenen wissenschaftlichen Kriterien vornehmen, beispielsweise wenn im Rahmen der Demokratiemessung Länderexpert*innen die Ausprägung dieser Kriterien einschätzen sollen. Allerdings wird diesen Expert*innen weder das Legitimitätsurteil überantwortet noch besteht ein analytisches Interesse an deren Urteil in ihrer Rolle als gesellschaftliche Akteur*innen. Vielmehr dienen die Expert*innen dazu, die Ausprägungen der definierten Indikatoren einschätzen zu können und sind somit extern beurteilende Akteur*innen. Solche Ansätze sind in der Tabelle unter Feld IX zu subsumieren. Die zweite Gruppe von Ansätzen sind solche, in denen aus theoretischen Debatten deduzierte Normen auf ihre gesellschaftliche Geltung und gegebenenfalls auch auf ihre Bedeutung für Legitimitätsurteile hin untersucht werden. Sie sind - auch wenn gesellschaftliche Normhorizonte nicht induktiv rekonstruiert werden - der mittleren Zeile der Tabelle zuzuordnen.

12 https://freedomhouse.org/report-types/freedom-world (Zugegriffen: 05. Juli 2019).

13 http://www.systemicpeace.org/polityproject.html (Zugegriffen: 05. Juli 2019).

14 https://www.demokratiematrix.de/ (Zugegriffen: 05. Juli 2019).
} 
der Debatte um das europäische Demokratiedefizit oft aktiv artikuliert und zum Teil auch mit Präskriptionen zur Überwindung des als defizitär attestierten Zustandes verbunden. So setzt Neyer (2010) Gerechtigkeit (nicht Demokratie) als normativen Maßstab an und unterbreitet aus dem urteilenden Abgleich von (normativer) Theorie und Empirie Vorschläge zur Überwindung der Differenz.

Jeweils liegt ein Kritikbegriff zugrunde, der die externe Definition eines normativen Maßstabs mit einer extern erfolgenden Abschätzung der Passung zwischen Theorie und Empirie verknüpft. Dies ähnelt einem Kritikbegriff, wie ihn Bittner bei Horkheimer attestiert, der darauf abhebe, ,den gegenwärtigen Zustand“ (Bittner 2009, S. 139) vor dem Maßstab des „,vernünftigen Zustands“ (Bittner 2009, S. 139), mithin also des normativ etablierten Ideals, zu bewerten. Das kritische Potenzial entfaltet eine solche normative Kritik aus der Diagnose des Abstandes der gesellschaftlichen Realität von einem (theoretisch) postulierten Idealzustand auf dessen Überwindung sie hinwirken kann: Deskription (der empirischen „Realität“) geschieht auch zum Zwecke der Emanzipation (vgl. Delanty 2011, S. 72; Horkheimer 1937, S. 277-278), wie es auch bei der Diagnose „,defekte[r] Demokratien“ (Merkel et al. 2003, S. 65) der Fall sein mag. ${ }^{15}$ Aus einer politikwissenschaftlichen Perspektive vertreten Gerring und Yesnowitz (2006, S. 101, 108, 133) eine Position, die sich von positivistischer Politikwissenschaft absetzen will und in der es Aufgabe der Wissenschaftler*innen ist, empirische Beobachtung normativ auszudeuten und zu bewerten - auch hier liegt die Wahl des Normhorizonts wie auch das zu treffende Urteil auf externer Ebene.

\subsection{Gesellschaftlicher Legitimitätsmaßstab, verschiedene Ebenen der Legitimitätsbeurteilung}

Die verbleibenden Felder in der mittleren Zeile der Tabelle beschreiben solche Ansätze, die das Werten intern verorten. Sie beziehen einen normativen Maßstab ein, der aus gesellschaftlichen Positionen, zu denen beispielsweise auch solche politischer Akteur*innen zählen, rekonstruiert ist. Zugleich stellen die Felder diejenigen dar, um die sich aktuell die stärkste Debatte hinsichtlich der Frage entfaltet, wie das interne Werten, also der interne normative Maßstab (und seine Konstruktion), forschungspraktisch erhoben werden kann (vgl. auch Ulbricht 2018, S. 1400). Diese gesellschaftlichen Normen beziehungsweise Legitimitätsverständnisse können induktiv erhoben werden. Sie können aber genauso zunächst theoretisch deduziert und dann auf ihre empirische Geltung hin abgetastet werden. Der interessierende Maßstab der Kritik bleibt in beiden Fällen ein gesellschaftlicher. ${ }^{16}$ Die Erhebung von gesellschaftlichen Legitimitätsmaßstäben erfordert also nicht zwingend einen

\footnotetext{
15 Die Frage nach legitimen Begründungen für normative Maßstäbe kann hier nicht diskutiert werden. Vgl. hierzu Dux und Globisch (2012), Dottori (2001), Jaeggi und Wesche (2009), Forst (2009). Angemerkt sei dennoch, dass an Horkheimer (und auch Adorno) kritisiert wird, die Substanz des vernünftigen Zustands und auch die Frage, wie dieser zu erkennen sei, bliebe ungeklärt (vgl. Bittner 2009, S. 140, 143; Delanty 2011, S. 72).

16 Durch diesen Einwand sollen nicht die Differenzen zwischen induktiver und deduktiver Forschungsanlage negiert werden. Vielmehr soll die Aufmerksamkeit auf die für das hier verfolgte Argument bedeutsamere Leitdifferenz gelenkt werden.
} 
induktiven Zugriff (vgl. beispielsweise Ahrens et al. 2011, S. 19-20). Vielmehr ist für die Einordnung der jeweiligen Forschungsbestrebungen weniger das forschungspraktische Vorgehen entscheidend, sondern wessen normativer Horizont als Maßstab herangezogen wird. Diese Ansätze mit einem Interesse für gesellschaftliche normative Legitimitätsstandards lassen sich wieder danach unterscheiden, ob und wenn ja, durch wen, ein auf diesem normativen Rahmen aufbauendes Legitimitätsurteil gefällt wird.

\subsubsection{Feld IV: internes Werten, keine Bewertung}

Ansätze in Feld IV rekonstruieren die normative Ordnung in Gesellschaften oder bestimmten gesellschaftlichen Segmenten. Hier steht zunächst nicht die Bewertung der Legitimität im Fokus, sondern die Existenz oder (Re-)Produktion sozialer Normen (vgl. Thornhill 2011, S. 135; Gaus 2011, S. 6-7, 9-10).

Beispielsweise sind Ansätze zu subsumieren, die sich auf die Identifikation von Demokratievorstellungen richten. Ihr Ziel ist es, zu erheben, ,wie Demokratie [...] begriffen wird" (Schubert und Weiß 2016b, S. 16, Hervorhebung dort). Die Forschungsrichtung reagiert nicht zuletzt auf das (vordergründige) Paradox, dass in weiten Teilen der Welt Demokratie als Wert zwar befürwortet wird, zugleich aber nichtdemokratische Herrschaftspraktiken Anerkennung finden (vgl. Schubert und Weiß 2016b, S. 10; Welzel und Kirsch 2017, S. 2). Dies wird über die Rekonstruktion von Demokratieverständnissen oder -vorstellungen eingehegt, indem gezeigt wird, dass Demokratievorstellungen häufig (aus westlicher Perspektive) nichtdemokratische Praktiken als Merkmal von Demokratiehaftigkeit umfassen. Welzel und Kirsch (2017) decken anhand von Umfragedaten auf, dass Bürger*innen - insbesondere in nicht- oder semidemokratischen Staaten - häufig ein autoritäres Verständnis von Demokratie vertreten. ${ }^{17}$ Letztlich zielen diese Ansätze - vermittelt über Demokratieverständnisse - auf die Rekonstruktion gesellschaftlicher Normstandards legitimen Regierens, sodass es sich vielleicht schlussendlich weniger um Demokratie-, denn um Legitimitätsverständnisse handelt.

Viele dieser Studien sind quantitativ ausgerichtet und nutzen Survey-Daten, wie des World Value Survey oder des European Social Survey (beispielsweise Welzel und Kirsch 2017; Ulbricht 2018; Fuchs und Roller 2016; Pickel 2016; Cho 2015; Shin 2015). Sie unterscheiden sich von den Ansätzen in Feld II dahingehend, dass sie sich nicht für die Urteile, sondern für deren normative Substanz interessieren. Obwohl das Gros der Studien mit quantitativen Daten und geschlossen Fragen arbeitet, gibt es einige Ansätze, die in diese large $n$-Analysen offene Fragen integrieren (beispielsweise Shin 2015; Dalton et al. 2007) oder qualitativen Befragungsdesigns mit einer geringeren Fallzahl und einem induktiven Zugriff zuneigen (beispielsweise Osterberg-Kaufmann 2016).

\footnotetext{
17 Hieraus schließen die Autor*innen, die Befragten würden „Demokratie missverstehen“ (Welzel und Kirsch 2017, S. 25, Übersetzung der Autorin), zumindest gegenüber dem normativen Demokratieideal der Autor*innen. Hier ist eine implizite Bewertung präsent, die solchen Ansätzen allerdings nicht innewohnen muss. Zudem handelt es sich um eine normative Bewertung des Demokratieverständnisses (auf das es hier nicht ankommt), nicht der Legitimität der politischen Ordnung.
} 
Der Forschungsstrang ist nicht auf die Nutzung von Umfragedaten beschränkt, sondern es werden etwa auch diskursanalytische Perspektiven eingenommen (vgl. beispielsweise die entsprechenden Beiträge in Schubert und Weiß 2016a). Hausteiner (2016) analysiert russische Demokratieverständnisse und zeigt, dass mit der Vorstellung der ,souveränen Demokratie“ (Hausteiner 2016, S. 171) und ,neo-eurasianistische[n]“ (Hausteiner 2016, S. 176) Konzepten zwei Demokratiekonzeptionen dominant sind, die sich gegen jene des Westens abgrenzen (vgl. Hausteiner 2016, S. 181). Eisentraut (2016) untersucht mittels Konkordanzanalyse das Demokratieverständnis politischer Akteur*innen hinsichtlich der Vereinten Nationen und arbeitet heraus, welche Konzepte mit Demokratie verbunden werden. Der rekonstruierte Normhorizont muss also nicht zwingend jener der Herrschaftsunterworfenen sein, sondern kann sich (wie in allen anderen internen Feldern) ebenso auf politische Eliten richten. So fokussiert auch Barnickel (2019) auf den Wandel der Normfundierung in der Legitimation der politischen Ordnung durch politische Eliten.

\subsubsection{Feld V: internes Werten, internes Bewerten}

Die vorrangig rekonstruktive Perspektive auf gesellschaftliche Normen ergänzen die Ansätze in Feld V um die Einbeziehung gesellschaftlicher Legitimitätsurteile. Sie sprechen zu der weiter oben formulierten Beschränkung in Patbergs Beurteilungsmodell, das die Bewertung der Legitimität von der internen Akteur*innen- auf die externe Beobachter*innenperspektive verschiebt. Hier werden nun beide Prozesse Werten und Bewerten - intern verortet und sowohl der normative Standard als auch die Beurteilung der gesellschaftlichen Akteur*innen erhoben. So akzentuiert Westle (2007, S. 115) aus der Perspektive der Umfrageforschung grundsätzlich, dass die Beurteilung des Systems (hier das Ausmaß an Demokratiezufriedenheit) von den normativen Erwartungen der Bürger*innen abhängt. Dies hatten bereits klassische Theorien der Legitimationsforschung betont. So unterstreicht Lipset (1983[1959], S. 64), dass die Legitimitätsbeurteilung gesellschaftlicher Gruppen von der Erfüllung ihrer normativen Erwartungen durch das politische System abhängt. Ähnlich verweist Easton (1975, S. 451) auf die Abhängigkeit der Evaluation von Objekten als legitim von der Konformität mit den der Evaluation zugrunde gelegten moralischen Prinzipien.

Gegenüber Easton und Lipset akzentuieren Boltanski und Thévenot (2014), denen es ebenfalls um die gesellschaftliche Normfundierung gesellschaftlicher Legitimitätsurteile geht, die gesellschaftlichen Praktiken der Normherstellung, mithin die Konstruktion des normativen Maßstabs. Sie eint nichtsdestotrotz das Interesse für gesellschaftliche Normativität und Kritik, wenngleich bei Easton und Lipset die evaluative Dimension deutlicher im Fokus stehen mag, während Boltanski und Thévenot vergleichsweise stärker den wertenden Aspekt der Normproduktion betonen.

Boltanski und Thévenot entwerfen in Über die Rechtfertigung eine Mikroperspektive auf Kritikprozesse. Sie verweisen darauf, dass in unterschiedlichen Rechtfertigungsordnungen verschiedene Bewertungsmaßstäbe relevant sind. Auf deren Basis werden in alltäglichen Situationen (insbesondere durch gesellschaftliche Akteur*innen) Evaluationen, Urteile, gefällt (vgl. Boltanski und Thévenot 2014, S. 32, 222-286, 1999, S. 368; Boltanski 2010, S. 47). Die Betrachtung der gesellschaftsin- 
ternen Kritik durch Akteur*innen des Alltags bleibt nicht auf die evaluative Komponente beschränkt. Vielmehr geht es ihnen ,zunächst [um] die Klärung der normativen Positionen, auf die die Akteure sich beim Kritisieren oder aber beim Rechtfertigen angesichts einer Kritik stützen können“"(Boltanski 2010, S. 48), was auf Prozesse der Herstellung von Bewertungskategorien verweist (vgl. Boltanski 2010, S. 41, 45-46).

Eine solche Perspektive wurde verschiedentlich aufgegriffen, modifiziert oder zumindest mit ihr sympathisiert (vgl. beispielsweise - ohne weiter auf die zweifelsohne bestehenden Unterschiede einzugehen - Vobruba 2013, S. 160-165). Dammayr et al. (2015, S. 11) attestieren der Legitimationsforschung insgesamt eine Hinwendung zu alltäglichen Praktiken der Erzeugung von Legitimität, die Arbeiten von Gadinger und Yildiz (2012) und Yildiz et al. (2018) knüpfen explizit an Boltanski und Thévenot an.

Sie schlagen einen diskursiv-narrativen Ansatz vor, der einbezieht, dass in diskursiven Aushandlungsprozessen ,,mittels kulturell verfügbarer Rechtfertigungsordnungen die normativen Ansprüche der Akteure verglichen werden" (Gadinger und Yildiz 2012, S. 308). Legitimität wird das temporäre Resultat von reflexiven Aushandlungsprozessen des adäquaten Rechtfertigungsmaßstabs und die Fähigkeit der Akteur*innen, sowohl zu werten als auch zu bewerten, einbezogen (vgl. Gadinger und Yildiz 2012, S. 303-304, 308-309, 312, 317). In der narrativen Perspektive schwingt die Frage nach der normativen Grundlage somit immer mit (vgl. Yildiz et al. 2018, S. 135).

Dies ist auch im bei Nullmeier et al. (2010) vertretenen Bewertungsmodell der Fall, welches Legitimationsaussagen verschiedener Akteur*innen (wie Journalist*innen, Zivilgesellschaft, Politiker*innen) inhaltsanalytisch sowohl nach der Bewertung der Legitimität als auch nach der der Bewertung zugrunde gelegten Norm absucht (vgl. Nullmeier und Nonhoff 2010, S. 16; Schmidtke und Nullmeier 2011, S. 124-128). Anwendung findet diese Herangehensweise in dem Sammelband Prekäre Legitimitäten (Nullmeier et al. 2010), in welchem öffentliche Debatten zur Legitimität mehrerer Nationalstaaten sowie inter- und supranationaler Organisationen untersucht werden, sowie in dem Band Marktwirtschaft in der Legitimationskrise (Nullmeier et al. 2014), der nach der öffentlichen Verhandlung der Legitimität der marktwirtschaftlichen Ordnung fragt (vgl. auch Haunss et al. 2015). Auch Autor*innen, die nicht dem Bremer Kreis, aus dem diese Forschungen hervorgegangen sind, zuzurechnen sind, bedienen sich des Instrumentariums. Binder und Heupel (2015) etwa analysieren in der Generalversammlung der Vereinten Nationen artikulierte Legitimationsaussagen hinsichtlich der Legitimität des Sicherheitsrates und zeigen auf, wie dessen Legitimität bewertet wird und welche Normen für die Bewertung herangezogen werden.

Obwohl die Mehrzahl der bisher genannten Studien einer inhalts- oder diskursanalytischen Vorgehensweise zuzurechnen ist, sind auch andere methodologische Zugriffe möglich: So zeigen Pickel und Jacobs (2002, S. 143, 145) - in ihrem Forschungsinteresse in der Tradition Eastons und anderer stehend und die Relevanz subjektiver Legitimität für Stabilität akzentuierend - anhand von Einstellungsdaten die Bedeutung (der Gewährleistung) von Rechten als Wertungsmaßstab für subjektive Legitimitätsurteile der Bürger*innen in Transformationsstaaten Mittelosteuropas. 


\subsubsection{Feld VI: internes Werten, externes Bewerten}

In Feld VI sind Ansätze verortet, die dem Beurteilungsmodell bei Patberg entsprechen. Hier wird der normative Standard der Bürger*innen als Grundlage des Wertens einbezogen, die Bewertung der Legitimität hingegen auf wissenschaftlicher Ebene vorgenommen. Patberg (2013, S. 158-160) hatte zur Umsetzung vorgeschlagen, die normativen Standards der Bürger*innen zu identifizieren (Werten), um sie dann mit der ,Realität“ der politischen Ordnung abzugleichen und aus dem Grad der Übereinstimmung das Legitimitätsurteil zu fällen (Bewerten). Auf diese Weise identifiziert - anknüpfend an die Debatte um das Legitimitätsdefizit der Europäischen Union Ehin (2008), welche normativen Erwartungen Bürger*innen gegenüber der europäischen Ordnung haben, um sodann der Europäischen Union zu diesem Zeitpunkt Legitimität abzusprechen, da sie nicht den liberal-demokratischen Erwartungen der Bürger*innen entspreche (vgl. Ehin 2008, S. 634). Die Beurteilung der Legitimität der Europäischen Union wird auf wissenschaftlicher Ebene vorgenommen, das Urteil basiert auf dem Abgleich mit gesellschaftlichen normativen Erwartungen.

Ähnlich verlagern sich spätere Generationen der Kritischen Theorie in dieses Feld. So weist Honneth darauf hin, dass der externe Hinweis ,,auf [...] das nicht ausgeschöpfte normative Potenzial“" (Boltanski und Honneth 2009, S. 100) auf einem normativen Rahmen basiert, der sich aus der normativen Ordnung der Gesellschaft selbst ergibt (vgl. Boltanski und Honneth 2009, S. 98-100). Aufgabe der Wissenschaftler*innen ist dann die Identifikation des Abstandes zwischen der normativen Ordnung der Gesellschaft und deren Realisation.

\section{Diskussion: (Meta-)Kritik in der Legitimationsforschung}

Durch die Übertragung der bewertungssoziologischen Differenzierung zwischen Werten und Bewerten sowie zwischen Normativität und Kritik werden unterschiedliche normative Lagerungen der Legitimationsforschung greifbar. Verortungen erfolgen danach, wessen normativer Referenzrahmen die Basis eines Legitimitätsurteils bildet und auf welcher Ebene - intern durch gesellschaftliche Akteur*innen oder extern durch wissenschaftliche Beobachter*innen - Legitimität beurteilt wird. Mit der Diskussion dieser Differenzierungen und der Illustration, dass diese gewinnbringend auf die Legitimationsforschung übertragen werden können, wurde eine Systematisierung vorgeschlagen, die diese grundlegenden Differenzierungen berücksichtigt und somit unterschiedliche Spielarten der (empirischen) Legitimationsforschung genauer als bisher verorten kann. So wie unterschiedliche Zugänge und Fokusse der Kritik als Ergänzung angesehen werden können (vgl. Delanty 2011, S. 88-89), sollte auch in der Legitimationsforschung der Mehrwert der gegenseitigen Befruchtung unterschiedlicher Ansätze unterstrichen werden. Insofern ist der vorliegende Text nicht als Plädoyer für die eine oder andere Forschungsrichtung zu betrachten, sondern hat systematisch die unterschiedliche normative Lagerung auch in der empirischen Forschung gezeigt und damit auch, dass je unterschiedliche Aspekte des Phänomens „Legitimität" in den Blick genommen werden. 
Was sind aber die Implikationen der vielfältigen Betrachtungsweisen für das Selbstverständnis der Sozial- und Politikwissenschaft und insbesondere für die Normativität der wissenschaftlichen Perspektive? Patberg, dessen Modelle des Messens und Beurteilens vorgestellt wurden, scheint nicht zuletzt die Sorge umzutreiben, dass sich die Sozialwissenschaften einer kritischen Perspektive entledigen (vgl. Patberg 2013, S. 163, 170; ähnlich auch Zürn 2011, S. 607). Einerseits ist die Sorge nachvollziehbar, wenn sowohl die Definition des normativen Ideals als auch die Bewertung auf die gesellschaftliche Ebene verlagert werden und die Aufgabe der Forschung auf die Rekonstruktion beider beschränkt ist. Denn selbstverständlich muss das, was von den Akteur*innen als anerkennungswürdig konstruiert wird, vor einem anderen normativen Maßstab nicht zwingend als normativ angemessen akzeptiert werden.

Hier ist einzuwenden, dass es einer kritischen Politikwissenschaft unbenommen bleibt, eine metakritische Perspektive einzunehmen. Diese kann sich auf rekonstruierte normative Maßstäbe beziehen und diese ihrerseits einer normativen Kritik unterwerfen. Dies kann ein erneutes Messen an einem zu begründenden Ideal umfassen. In dem Fall hätten wir es mit einer doppelten Normativität zu tun: Der Normativität der (politischen und gesellschaftlichen) Akteur*innen in ihren Versuchen der Herstellung von Legitimität und einer zweiten, externen, normativen Perspektive, die diese Versuche - vor einem möglicherweise abweichenden normativen Maßstab einordnet (ähnlich Patberg 2013, S. 160, 163, 170; Barnickel 2019, S. 28-29; für ein empirisches Beispiel der extern-normativen Kritik von rekonstruierten Rechtfertigungsnarrativen vgl. Lehner 2015). Wie dieser Maßstab aussehen kann, ist wiederum normativ zu begründen. Eine alternative Vorgehensweise ist mit Rahel Jaeggi (2009) denkbar. Ihr Entwurf einer Ideologiekritik verzichtet zwar auf einen externen Maßstab, will sich aber gleichzeitig nicht den internen Maßstab zu eigen machen (vgl. insbesondere Jaeggi 2009, S. 267), sondern ,,aus den Selbstwidersprüchen der gegebenen Normen und der gegebenen Realität die Maßstäbe zu deren Überwindung [generieren]“ (Jaeggi 2009, S. 284, Hervorhebung hinzugefügt).

Eine durch Foucault inspirierte kritische Perspektive würde demgegenüber weniger in einer Kritik der Substanz der normativen Horizonte (wie sie in Feld IV bis VI rekonstruiert werden) und „falschen Idee[n]“ (Foucault 1992, S. 30) liegen, sondern vielmehr in der Offenlegung von Bedingungen und Kontingenzen des Wissens, des So-Seins rekonstruierter normativer Ordnungen und ihren Verbindungen zu Macht (vgl. Foucault 1992, S. 32-34, 46, 49). Eine solche Sichtweise erfordert dann nicht nur eine Offenlegung der Kontingenzen gesellschaftlicher Wissensordnungen, sondern auch eine Befragung der wissenschaftlichen Normativität und ihren Wertungsordnungen auf ihre Verwebung mit Macht-Wissens-Komplexen und somit eine kritische Reflexion der Positionalität der Wissenschaftler*innen und der Wissenschaft selbst. Eine solchermaßen metakritische Position müsste sich dann einerseits mit der Frage auseinandersetzen, wie (nicht nur gesellschaftliche) legitimatorische Wertungsordnungen produziert und stabilisiert werden. Andererseits hätte sie neben der eigenen Verstrickung in normative Wissensordnungen ihre Rolle in der Prägung, (Re-)Produktion und/oder Stabilisierung (öffentlicher) Diskurse und Wissens- und Wertungsordnungen zu hinterfragen. 
Danksagung Für wertvolle und detaillierte Kommentare zum eingereichten Manuskript danke ich den anonymen Gutachter*innen sowie der Redaktion der PVS. Frühere Versionen des Textes wurden im Kolloquium des Viadrina Instituts für Europastudien sowie auf dem Kongress der Deutschen Vereinigung für Politkwisssenschaft diskutiert. Für hilfreiche Hinweise danke ich den jweiligen Teilnehmer*innen, insbesondere Christoph Mohamad-Klotzbach. Inspirierende Diskussionen zu Kritikbegriffen habe ich mit den Teilnehmer*innen des Lektürekreises „Europa und Kritik“ am Viadrina Institut für Europastudien geführt.

Funding Open Access funding enabled and organized by Projekt DEAL.

Open Access Dieser Artikel wird unter der Creative Commons Namensnennung 4.0 International Lizenz veröffentlicht, welche die Nutzung, Vervielfältigung, Bearbeitung, Verbreitung und Wiedergabe in jeglichem Medium und Format erlaubt, sofern Sie den/die ursprünglichen Autor(en) und die Quelle ordnungsgemäß nennen, einen Link zur Creative Commons Lizenz beifügen und angeben, ob Änderungen vorgenommen wurden.

Die in diesem Artikel enthaltenen Bilder und sonstiges Drittmaterial unterliegen ebenfalls der genannten Creative Commons Lizenz, sofern sich aus der Abbildungslegende nichts anderes ergibt. Sofern das betreffende Material nicht unter der genannten Creative Commons Lizenz steht und die betreffende Handlung nicht nach gesetzlichen Vorschriften erlaubt ist, ist für die oben aufgeführten Weiterverwendungen des Materials die Einwilligung des jeweiligen Rechteinhabers einzuholen.

Weitere Details zur Lizenz entnehmen Sie bitte der Lizenzinformation auf http://creativecommons.org/ licenses/by/4.0/deed.de.

\section{Literatur}

Aarts, Kees, Carolien van Ham, und Jacques Thomassen. 2017. Modernization, globalization, and satisfaction with democracy. In Myth and reality of the legitimacy crisis. Explaining trends and crossnational differences in established democracies, Hrsg. Carolien van Ham, Jacques Thomassen, Kees Aarts, und Rudy B. Andeweg, 37-58. Oxford: Oxford University Press.

Abromeit, Heidrun, und Michael Stoiber. 2007. Criteria of democratic legitimacy. In Legitimacy in an age of global politics, Hrsg. Achim Hurrelmann, Steffen Schneider, und Jens Steffek, 35-56. Houndmills: Palgrave Macmillan.

Ahrens, Johannes, Raphael Beer, Uwe H. Bittlingmayer, und Jürgen Gerdes. 2011. Normativität. Über die Hintergründe sozialwissenschaftlicher Theoriebildung. Zur Einführung. In Normativität. Über die Hintergründe sozialwissenschaftlicher Theoriebildung, Hrsg. Johannes Ahrens, Raphael Beer, Uwe H. Bittlingmayer, und Jürgen Gerdes, 9-24. Wiesbaden: VS.

Allerkamp, Andrea, Pablo Valdivia Orozco, und Sophie Witt. 2015. Einleitung. Zum Gegen/Stand der Kritik. In Gegen/Stand der Kritik, Hrsg. Andrea Allerkamp, Pablo Valdivia Orozco, und Sophie Witt, 7-26. Zürich, Berlin: Diaphanes.

Baran, Pavel, und Heinz Wagner. 1990. Norm. In Europäische Enzyklopädie zu Philosophie und Wissenschaften, Bd. 3, Hrsg. Hans J. Sandkühler, Arnim Regenbogen, Chup Friemert, Werner Goldschmidt, Lars Lembrecht, Thomas Mies, Detlev Pätzold, und Heinz Wagner, 573-584. Hamburg: Meiner.

Barker, Rodney. 2007. Democratic legitimation: what is it, who wants it, and why? In Legitimacy in an age of global politics, Hrsg. Achim Hurrelmann, Steffen Schneider, und Jens Steffek, 19-33. Houndmills: Palgrave Macmillan.

Barnickel, Christiane. 2019. Postdemokratisierung der Legitimationspolitik. Diskursnetzwerke in bundesdeutschen Großen Regierungserklärungen und Aussprachen 1949-2014. Wiesbaden: Springer VS.

Beetham, David, und Lord Christopher. 1998. Legitimacy and the EU. London: Longman.

Bein, Simon. 2018. Demokratien in der Krise? Zum Beitrag zentraler Indizes der Demokratiemessung zur Debatte um Funktionsstörungen etablierter Demokratien. Zeitschrift für Politikwissenschaft 28:147-171.

Binder, Martin, und Monika Heupel. 2015. The legitimacy of the UN security council. Evidence from recent general assembly debates. International Studies Quarterly 59:238-250.

Bittner, Rüdiger. 2009. Kritik, und wie es besser wäre. In Was ist Kritik?, Hrsg. Rahel Jaeggi, Tilo Wesche, 134-149. Frankfurt am Main: Suhrkamp.

Boltanski, Luc. 2010. Soziologie und Sozialkritik. Berlin: Suhrkamp. 
Boltanski, Luc, und Axel Honneth. 2009. Soziologie der Kritik oder Kritische Soziologie? Ein Gespräch mit Robin Celikates. In Was ist Kritik?, Hrsg. Rahel Jaeggi, Tilo Wesche, 81-114. Frankfurt am Main: Suhrkamp.

Boltanski, Luc, und Laurent Thévenot. 1999. The sociology of critical capacity. European Journal of Social Theory 2:359-377.

Boltanski, Luc, und Laurent Thévenot. 2014. Über die Rechtfertigung. Eine Soziologie der kritischen Urteilskraft. Hamburg: Hamburger Edition.

Bühlmann, Marc, Wolfgang Merkel, Lisa Müller, Heiko Giebler, und Bernhard Weßels. 2012. Demokratiebarometer. Ein neues Instrument zur Messung von Demokratiequalität. Zeitschrift für Vergleichende Politikwissenschaft 6:115-159.

Bürgler, Beatrice. 2009. Demokratische Legitimität in der internationalen Umweltpolitik. Wiesbaden: VS.

Campbell, David F.J., und Thorsten D. Barth. 2009. Wie können Demokratie und Demokratiequalität gemessen werden? Modelle, Demokratie-Indices und Länderbeispiele im globalen Vergleich. SWSRundschau 49:209-233.

Cho, Youngho. 2015. How well are global citizenries informed about democracy? Ascertaining the breadth and distribution of their democratic enlightenment and its sources. Political Studies 63:240-258.

Cohen, Joshua. 1989. Deliberation and Democratic Legitimacy. In The good polity. Normative analysis of the state, Hrsg. Alan P. Hamlin, Philip Pettit, 17-34. Oxford: Basil Blackwell.

Dalton, Russell J., C. Shin Doh, und Willy Jou. 2007. Understanding democracy: data from unlikely places. Journal of Democracy 18:142-156.

Dammayr, Maria, Doris Graß, und Barbara Rothmüller. 2015. Legitimität und Legitimierung in der sozialwissenschaftlichen Debatte: eine Einführung in die Theorien der Rechtfertigung und Kritik von Herrschaft. In Legitimität. Gesellschaftliche, politische und wissenschaftliche Bruchlinien der Rechtfertigung, Hrsg. Maria Dammayr, Doris Graß, und Barbara Rothmüller, 7-24. Bielefeld: transcript.

Delanty, Gerard. 2011. Varieties of critique in sociological theory and their methodological implications for social research. Irish Journal of Sociology 19:68-92.

Dellmuth, Lisa M., und Jonas Tallberg. 2015. The social legitimacy of international organisations. Interest representation, institutional performance, and confidence extrapolation in the United Nations. Review of International Studies 41:451-475.

Dlabac, Oliver, und Hans-Peter Schaub. 2012. Ein duales Messkonzept für liberale und radikale Demokratiequalität. Zeitschrift für Vergleichende Politikwissenschaft 6:161-184.

Dottori, Riccardo. 2001. Normativity and legitimacy. Introduction to the meeting. In Normativity and legitimacy. Normatività e Legittimazione. Proceedings of the II Meeting. Italian-American Philosophy-New York 1999., Hrsg. Riccardo Dottori, 11-28. Münster: LIT.

Dux, Günther, und Claudia Globisch. 2012. Soziologie der Kritik. Soziologische Revue 35:417-428.

Easton, David. 1965. A systems analysis of political life. New York, London, Sidney: John Wiley \& Sons.

Easton, David. 1975. A re-assessment of the concept of political support. British Journal of Political Science 5:435-457.

Ehin, Piret. 2008. Competing models of EU legitimacy: the test of popular expectations. Journal of Common Market Studies 46:619-640.

Eisentraut, Sophie. 2016. Demokratisches Regieren jenseits des Nationalstaats: Demokratiekonzeptionen aus sechs Weltregionen. In „Demokratie“ jenseits des Westens. Theorien, Diskurse, Einstellungen, Hrsg. Sophia Schubert, Alexander Weiß, 147-166. Baden-Baden: Nomos.

Eriksen, Erik O., und John Erik Fossum. 2004. Europe in search of legitimacy: strategies of legitimation assessed. International Political Science Review 25:435-459.

Føllesdal, Andreas. 2006. Survey article: the legitimacy deficits of the European Union. The Journal of Political Philosophy 14:441-468.

Forst, Rainer. 2009. Der Grund der Kritik. Zum Begriff der Menschenwürde in sozialen Rechtfertigungsordnungen. In Was ist Kritik?, Hrsg. Rahel Jaeggi, Tilo Wesche, 150-164. Frankfurt am Main: Suhrkamp.

Foucault, Michel. 1992. Was ist Kritik? Berlin: Merve.

Fuchs, Dieter. 2000. Demos und Nation in der Europäischen Union. In Zur Zukunft der Demokratie. Herausforderungen im Zeitalter der Globalisierung (WZB-Jahrbuch), Hrsg. Hans-Dieter Klingemann, Friedhelm Neidhardt, 215-236. Berlin: Rainer Bohn.

Fuchs, Dieter. 2011. Cultural diversity, European identity and legitimacy of the EU: a theoretical framework. In Cultural diversity, European identity and the legitimacy of the EU, Hrsg. Dieter Fuchs, HansDieter Klingemann, 27-57. Cheltenham: Edward Elgar.

Fuchs, Dieter, und Edeltraud Roller. 2016. Demokratiekonzeptionen der Bürger und demokratische Gemeinschaftsorientierungen. Westliche, ostasiatische und arabische Länder im Vergleich. In „Demo- 
kratie“ jenseits des Westens. Theorien, Diskurse, Einstellungen, Hrsg. Sophia Schubert, Alexander Weiß, 296-317. Baden-Baden: Nomos.

Gadinger, Frank, und Taylan Yildiz. 2012. Unruhige Legitimität. Die Finanzkrise aus Sicht der pragmatischen Soziologie der Kritik. In Der Aufstieg der Legitimitätspolitik. Rechtfertigung und Kritik politisch-ökonomischer Ordnungen. Leviathan Sonderheft 27/2012. Hrsg. Anna Geis, Frank Nullmeier, und Christopher Daase, 302-318. Baden-Baden: Nomos.

Gaus, Daniel. 2011. The dynamics of legitimation. Why the study of political legitimacy needs more realism. RECON Online Working Paper 2011/15. Oslo: Arena. www.reconproject.eu/main.php/RECON_wp_ 1115.pdf?fileitem=50512026 (Zugegriffen: 31. Dezember 2017).

Gerring, John, und Joshua Yesnowitz. 2006. A normative turn in political science? Polity 38:101-133.

Gilley, Bruce. 2012. State legitimacy: an updated dataset for 52 countries. European Journal of Political Research 51:693-699.

Habermas, Jürgen. 1976. Legitimationsprobleme im modernen Staat. In Legitimationsprobleme politischer Systeme, Hrsg. Peter G. Kielmansegg, 39-61. Opladen: Westdeutscher Verlag.

Habermas, Jürgen. 1996. Die Einbeziehung des Anderen. Studien zur politischen Theorie. Frankfurt am Main: Suhrkamp.

Haunss, Sebastian, Falk Lenke, Henning Schmidtke, und Steffen Schneider. 2015. Finanzkrise ohne Legitimationskrise? Kapitalismuskritik in der deutschen Qualitätspresse. In Legitimität. Gesellschaftliche, politische und wissenschaftliche Bruchlinien der Rechtfertigung, Hrsg. Maria Dammayr, Doris Graß, und Barbara Rothmüller, 73-94. Bielefeld: transcript.

Hausteiner, Eva M. 2016. Demokratie als Provokation: Russische Demokratiebegriffe zwischen Theorie und Propaganda. In „Demokratie“ jenseits des Westens. Theorien, Diskurse, Einstellungen, Hrsg. Sophia Schubert, Alexander Weiß, 167-187. Baden-Baden: Nomos.

Horkheimer, Max. 1937. Traditionelle und kritische Theorie. Zeitschrift für Sozialforschung 6:245-294.

Horkheimer, Max, und Herbert Marcuse. 1937. Philosophie und kritische Theorie. Zeitschrift für Sozialforschung 6:625-647.

Jaeggi, Rahel. 2009. Was ist Ideologiekritik? In Was ist Kritik?, Hrsg. Rahel Jaeggi, Tilo Wesche, 266-295. Frankfurt am Main: Suhrkamp.

Jaeggi, Rahel, und Tilo Wesche. 2009. Einführung: Was ist Kritik? In Was ist Kritik?, Hrsg. Rahel Jaeggi, Tilo Wesche, 7-20. Frankfurt am Main: Suhrkamp.

Kielmansegg, Peter G. 2003. Integration und Demokratie. In Europäische Integration, Hrsg. Markus Jachtenfuchs, Beate Kohler-Koch, 49-83. Opladen: Leske + Budrich.

Kriesi, Hanspeter. 2013. Democratic legitimacy: is there a legitimacy crisis in contemporary politics? Politische Vierteljahresschrift 54:609-638.

Krüger, Anne K., und Martin Reinhart. 2016. Wert, Werte und (Be)Wertungen. Eine erste begriffs- und prozesstheoretische Sondierung der aktuellen Soziologie der Bewertung. Berliner Journal für Soziologie 26:485-500.

Lamont, Michèle. 2012. Toward a comparative sociology of valuation and evaluation. Annual Review of Sociology 38:201-221.

van Leeuwen, Theo. 2007. Legitimation in discourse and communication. Discourse \& Communication $1: 91-112$.

van Leeuwen, Theo, und Ruth Wodak. 1999. Legitimizing immigration control. A discourse-historical analysis. Discourse Studies 1:83-118.

Lehner, Daniel. 2015. Die Unangemessenheit des „demokratischen Versprechens“ - zur Brüchigkeit der politischen Rechtfertigungsordnung. In Legitimität. Gesellschaftliche, politische und wissenschaftliche Bruchlinien der Rechtfertigung, Hrsg. Maria Dammayr, Doris Graß, und Barbara Rothmüller, 27-48. Bielefeld: transcript.

Lembcke, Oliver W., Claudia Ritzi, und Gary S. Schaal (Hrsg.). 2012a. Normative Demokratietheorien. Zeitgenössische Demokratietheorie, Bd. 1. Wiesbaden: VS.

Lembcke, Oliver W., Claudia Ritzi, und Gary S. Schaal. 2012b. Zwischen Konkurrenz und Konvergenz. Eine Einführung in die normative Demokratietheorie. In Normative Demokratietheorien Zeitgenössische Demokratietheorie, Bd. 1, Hrsg. Oliver W. Lembcke, Claudia Ritzi, und Gary S. Schaal, 9-32. Wiesbaden: VS.

Lipset, Seymour M. 1983 [1959]. Political man. The social bases of politics. London: Heinemann.

Lübbe, Weyma. 1991. Legitimität kraft Legalität: Sinnverstehen und Institutionenanalyse bei Max Weber und seinen Kritikern. Tübingen: Mohr Siebeck.

Luckmann, Thomas. 1987. Comments on legitimation. Current Sociology 35:109-117.

Luhmann, Niklas. 2008 [1969]. Legitimation durch Verfahren. Frankfurt am Main: Suhrkamp. 
Merkel, Wolfgang, Hans-Jürgen Puhle, Aurel Croissant, Claudia Eicher, und Peter Thiery. 2003. Defekte Demokratie. Band 1: Theorie. Opladen: Leske + Budrich.

Neyer, Jürgen. 2010. Justice, not democracy: legitimacy in the European Union. Journal of Common Market Studies 48:903-921.

Norris, Pippa. 1999. Critical citizens. Global support for democratic government. Oxford: Oxford University Press.

Nullmeier, Frank, und Martin Nonhoff. 2010. Der Wandel des Legitimitätsdenkens. In Prekäre Legitimitäten. Rechtfertigung von Herrschaft in der postnationalen Konstellation, Hrsg. Frank Nullmeier, Dominika Biegoń, Jennifer Gronau, Martin Nonhoff, Henning Schmidtke, und Steffen Schneider, 16-44. Frankfurt, New York: Campus.

Nullmeier, Frank, Dominika Biegoń, Jennifer Gronau, Martin Nonhoff, Henning Schmidtke, und Steffen Schneider (Hrsg.). 2010. Prekäre Legitimitäten. Rechtfertigung von Herrschaft in der postnationalen Konstellation. Frankfurt, New York: Campus.

Nullmeier, Frank, Dominika Biegoń, Jennifer Gronau, Sebastian Haunss, Falk Lenke, Henning Schmidtke, und Steffen Schneider (Hrsg.). 2014. Marktwirtschaft in der Legitimationskrise? Ein internationaler Vergleich. Frankfurt am Main, New York: Campus.

O'Neill, Onora. 2000. Starke und schwache Gesellschaftskritik in einer globalisierten Welt. Deutsche Zeitschrift für Philosophie 48:719-728.

Osterberg-Kaufmann, Norma. 2016. „How People View Democracy“: Messprobleme und mögliche Alternativen. In „Demokratie“ jenseits des Westens. Theorien, Diskurse, Einstellungen, Hrsg. Sophia Schubert, Alexander Weiß, 343-365. Baden-Baden: Nomos.

Patberg, Markus. 2013. Zwei Modelle empirischer Legitimitätsforschung - Eine Replik auf Michael Zürns Gastbeitrag in der PVS 4/2011. Politische Vierteljahresschrift 54:155-172.

Peter, Fabienne. 2009. Democratic legitimacy. New York: Routledge.

Pickel, Gert, und Jörg Jacobs. 2002. „Subjektive Legitimität“, Rechtsstaat und Konsolidierung. In Recht in der Transformation. Rechts- und Verfassungswandel in Mittel- und Osteuropa: Beiträge zur Debatte, Hrsg. Christian Boulanger, 125-148. Berlin: Berliner Debatte Wissenschaftsverlag.

Pickel, Susanne. 2016. Konzepte und Verständnisse von Demokratie in Ost- und Westeuropa. In „Demokratie“ jenseits des Westens. Theorien, Diskurse, Einstellungen, Hrsg. Sophia Schubert, Alexander Weiß, 318-342. Baden-Baden: Nomos.

Pickel, Susanne, und Gert Pickel. 2012. Die Messung von Indizes in der Vergleichenden Politikwissenschaft - methodologische Spitzfindigkeit oder substantielle Notwendigkeit. Zeitschrift für Vergleichende Politikwissenschaft 6:1-17.

Rojo, Luisa M., und Teun A. van Dijk. 1997. „There was a problem and it was solved!“: legitimating the expulsion of 'illegal' migrants in Spanish parliamentary discourse. Discourse \& Society 8:523-566.

Röttgers, Kurt. 1982. Kritik. In Geschichtliche Grundbegriffe. Historisches Lexikon zur politisch-sozialen Sprache in Deutschland, Bd. 3, Hrsg. Otto Brunner, Werner Conze, und Reinhart Koselleck, 651-675. Stuttgart: Klett-Cotta.

Scharpf, Fritz W. 1999. Regieren in Europa: effektiv und demokratisch. Frankfurt am Main, New York: Campus.

Schmidt, Vivien A. 2013. Democracy and legitimacy in the European Union revisited: input, output and throughput. Political Studies 61:2-22.

Schmidtke, Henning, und Frank Nullmeier. 2011. Political valuation analysis and the legitimacy of international organizations. German Policy Studies 7:117-153.

Schmidtke, Henning, und Steffen Schneider. 2012. Methoden der empirischen Legitimationsforschung: Legitimität als mehrdimensionales Konzept. In Der Aufstieg der Legitimitätspolitik. Rechtfertigung und Kritik politisch-ökonomischer Ordnungen. Leviathan Sonderheft 27/2012. Hrsg. Anna Geis, Frank Nullmeier, und Christopher Daase, 225-242. Baden-Baden: Nomos.

Schubert, Sophia, und Alexander Weiß (Hrsg.). 2016a. „Demokratie“ jenseits des Westens. Theorien, Diskurse, Einstellungen. Baden-Baden: Nomos.

Schubert, Sophia, und Alexander Weiß. 2016b. Demokratiekonzeptionen jenseits des Westens: Ein theoretischer Rahmen für ein neues Forschungsfeld. In „Demokratie“ jenseits des Westens. Theorien, Diskurse, Einstellungen, Hrsg. Sophia Schubert, Alexander Weiß, 6-34. Baden-Baden: Nomos.

Shin, Doh C. 2015. Assessing citizen responses to democracy: a review and synthesis of recent public opinion research. Center for the Study of Democracy Working Papers. Irvine: University of California.

Thornhill, Chris. 2011. Political legitimacy: a theoretical approach between facts and norms. Constellations 18:135-169.

Ulbricht, Tom. 2018. Perceptions and conceptions of democracy. Applying thick concepts of democracy to reassess desires for democracy. Comparative Political Studies 51:1387-1440. 
Vaara, Eero. 2014. Struggles over legitimacy in the Eurozone crisis. Discursive legitimation strategies and their ideological underpinnings. Discourse \& Society 25:500-518.

Vatin, François. 2013. Valuation as evaluating and valorizing. Valuation Studies 1:31-50.

Vobruba, Georg. 2013. Soziologie und Kritik. Moderne Sozialwissenschaft und Kritik der Gesellschaft. Soziologie 42:147-168.

Weber, Max. 1980 [1921]. Wirtschaft und Gesellschaft. Tübingen: J.C.B. Mohr.

Welzel, Christian, und Helen Kirsch. 2017. Democracy misunderstood: authoritarian notions of democracy around the globe. World Values Research 9:1-29.

Wesche, Tilo. 2009. Reflexion, Therapie, Darstellung. Formen der Kritik. In Was ist Kritik?, Hrsg. Rahel Jaeggi, Tilo Wesche, 193-220. Frankfurt am Main: Suhrkamp.

Westle, Bettina. 2007. Legitimacy in an age of global politics. In Political beliefs and attitudes: legitimacy in public opinion research, Hrsg. Achim Hurrelmann, Steffen Schneider, und Jens Steffek, 93-125. Houndmills: Palgrave Macmillan.

Yildiz, Taylan, Frank Gadinger, und Christopher Smith. 2018. Narrative Legitimierung: Exekutive, repräsentative und subversive Erzählstrategien in der Überwachungskontroverse. Leviathan 46:135-162.

Zürn, Michael. 2011. Perspektiven des demokratischen Regierens und die Rolle der Politikwissenschaft im 21. Jahrhundert. Politische Vierteljahresschrift 52:603-635.

Zürn, Michael. 2012. Autorität und Legitimität in der postnationalen Konstellation. In Der Aufstieg der Legitimitätspolitik. Rechtfertigung und Kritik politisch-ökonomischer Ordnungen. Leviathan Sonderheft 27/2012. Hrsg. Anna Geis, Frank Nullmeier, und Christopher Daase, 41-62. Baden-Baden: Nomos. 